\title{
Editorial: The Use of Virtual-Reality Interventions in Reducing Anxiety
}

\author{
Preethi Premkumar ${ }^{1 *}$, Nadja Heym ${ }^{2}$, Page Lyn Anderson ${ }^{3}$, David Brown ${ }^{4}$ and \\ Alexander Sumich ${ }^{2}$ \\ ${ }^{1}$ Division of Psychology, London South Bank University, London, United Kingdom, ${ }^{2}$ Department of Psychology, Nottingham Trent \\ University, Nottingham, United Kingdom, ${ }^{3}$ Department of Psychology, Neuroscience Institute, Georgia State University, Atlanta, \\ GA, United States, ${ }^{4}$ Department of Computer Science, Nottingham Trent University, Nottingham, United Kingdom
}

Keywords: attention training, presence, cybersickness, relaxation, pain

Editorial on the Research Topic

The Use of Virtual-Reality Interventions in Reducing Anxiety

\section{INTRODUCTION}

Virtual-reality (VR) therapy for anxiety disorders has emerged with the advent of VR technology. Its superior efficacy to waitlist and comparable efficacy to in vivo exposure therapy (Carl et al., 2019) make VR therapy a viable psychological intervention. VR therapy offers several accessibility benefits to encourage help-seeking among those who are less inclined to engage in face-to-face therapy and those with mild-to-moderate and/or sub-clinical levels of anxiety who might not reach threshold for clinical referral. This special issue aimed to understand the advantages and limits of VR therapy in improving symptoms of anxiety. Anxiety is the anticipation of real threat (Penninx et al., 2021;

OPEN ACCESS

Edited and reviewed by: Albert Rizzo, University of Southern California, United States

*Correspondence: Preethi Premkumar premkump@lsbu.ac.uk

Specialty section:

This article was submitted to Virtual Reality in Medicine, a section of the journal Frontiers in Virtual Reality

Received: 12 January 2022 Accepted: 14 January 2022 Published: 09 February 2022

Citation: Hamm, 2020). Anxiety is characterized by "muscle tension and vigilance in preparation for future danger and cautious avoidance behavior" (American Psychiatric Association, 2013). Anxiety includes a cognitive element, including a fear of the worst happening, and a physiological element, such as heightened arousal, sweating and feeling faint (Creamer et al., 1995). The objectives of this special issue were to understand:

- The theoretical significance of VR therapy for clinical and sub-clinical levels of anxiety,

- The efficacy of VR therapy for reducing anxiety,

- The mechanisms of response to VR therapy for reducing anxiety, and

- The technological limits of VR therapy.

\section{THEORETICAL UNDERPINNINGS OF VIRTUAL-REALITY THERAPY FOR ANXIETY}

Research on virtual reality for anxiety disorders must be guided by theory because the "wow" factor of the technology can lead people to develop virtual environments for clinical applications acontextually. An early randomized clinical trial used virtual reality for fear of heights (Rothbaum et al., 1995) and it relied on emotional processing theory (Foa and Kozak, 1986), which suggests that exposure therapy modulates a dysfunctional fear structure and provides information that is incompatible with the fear-associated memory. In this issue, Jerath and Beveridge draw on theories of the philosophy of mind and theories of global and spatial cognition to inspire future research on how virtual reality can improve anxiety through the 
creation of massive virtual spaces, such as looking at a clear sky at night. The authors suggest that anxiety is characterized by vicious cycles that can be interrupted by transcendent experiences created in expansive virtual spaces, like the cosmos, using slow deep breathing and biofeedback. Advances in VR technology to create massive spaces through testable hypotheses would yield exciting possibilities to investigate the benefits of transcendent virtual experiences.

\section{EFFICACY OF VIRTUAL-REALITY THERAPY}

VR technology holds promise for self-guided VR therapy where the user has full control over increasing their exposure to threat in the absence of a therapist (Zainal et al., 2021; Premkumar et al.). Participants with high self-reported public-speaking anxiety increased their exposure to the modifiable virtual threats over two sessions of self-guided VR therapy (Premkumar et al.). Findings of sustained improvement in public-speaking anxiety and social anxiety 1 month after the intervention in this preliminary study suggest the long-term benefits of self-guided VR therapy. Where anxiety is brought about by chronic physical illness, such as cancer, VR therapy can construct positive virtual environments. MIND (2021) note that "spending time in green space or bringing nature into your everyday life can benefit both your mental and physical wellbeing" (Naor and Mayseless, 2021). It is within this context that Wilson and Scorsone explored the benefits of bringing people closer to nature through immersive VR therapy and inducing positive emotions and reducing pain levels. Selecting among a wide range of natureinspired immersive audio-visual experiences to promote relaxation during treatment via chemotherapy, participants receiving intravenous chemotherapy felt more calm, relaxed, and content, as well as less tense, thereby improving adherence to treatment. This anxietyreducing impact of VR therapy during treatment for physical condition is true of other related conditions, such as stroke rehabilitation (Standen et al., 2017).

\section{MECHANISM OF VIRTUAL-REALITY THERAPY}

There is a need to understand more precisely the mechanisms of anxiety reduction that can be incorporated into VR therapies. To this end, Pfaller et al. highlighted the role of social presence in evoking emotional responses during specific agent social interactions. Thus, manipulating social presence may lead to more effective interventions in improving social interaction. A further mechanism of VR therapy is to gain control over anxious thoughts through attention training. Wechsler et al. examined the effect of external-focus attention training in participants with high public-speaking anxiety. Training participants to attend to members of the audience in the virtual environment increased the time they spent looking at the virtual audience during a posttraining public-speech and enhanced their positive affect. Thus, attending to a virtual core threat is a key mechanism of VR exposure therapy.

There are also physiological mechanisms that alter anxiety response. Joeng et al. examined the impact of diaphragmatic breathing (DB, increasing breathing volume and allowing more air to the body) and progressive muscle relaxation (PMR, sequentially alternating muscle tension and relaxation) exercises in a VR-based relaxation training program. Levels of tension after PMR were lower in those who trained in VR compared to a control group. DB decreased tension in the VR group, particularly when they practiced in virtual outdoor anxiety-provoking environments. DB primarily strengthens the parasympathetic response, whilst also improving sustained attention and decreasing negative affect (Ma et al., 2017). Thus, incorporating both physiological techniques as training elements to facilitate additional coping strategies, would aid VR-based anxiety exposure therapies.

\section{TECHNOLOGICAL UNDERPINNINGS OF VIRTUAL-REALITY THERAPY}

Cybersickness, such as dizziness and motion-sickness, affects the engagement in and experience of VR therapy. Controlling for cybersickness in research on VR therapy is necessary and it requires a validated measure of cybersickness that segregates cybersickness from the target psychological symptoms of the intended intervention. Besides confirming the two-factor structure of the simulation-sickness questionnaire (SSQ, study 1), Bouchard et al. also revealed that anxiety during stress-exposure (the Trier Social Stress Test, study 2) was confounded by cybersickness. They found that most items (11 out of 16) of the SSQ correlated with state anxiety during stress-exposure, and these correlations were split evenly across the two factors of the SSQ. Scores on the SSQ increased after stress exposure and before immersion in a virtual environment. Thus, cybersickness may be confused with anxiety during administration of VR therapy and such symptoms need to be controlled for when examining the efficacy of VR therapy.

This special issue has revealed the potential for theoreticallymeaningful innovations in VR therapy. The scope for novel VR therapies to integrate virtual wide open spaces, self-guided exposure and relaxation, whilst enhancing social presence through attention training and addressing cybersickness demonstrates the immense possibilities of virtual reality.

\section{AUTHOR CONTRIBUTIONS}

Each author wrote a different section of the article by summarizing an article from the special issue. All authors contributed to proof-reading the article. 


\section{REFERENCES}

American Psychiatric Association (2013). Diagnostic and Statistical Manual of Mental Disorders: DSM-5. Arlington, VA, US: American Psychiatric Publishing, Inc.

Carl, E., Stein, A. T., Levihn-Coon, A., Pogue, J. R., Rothbaum, B., Emmelkamp, P., et al. (2019). Virtual Reality Exposure Therapy for Anxiety and Related Disorders: A Meta-Analysis of Randomized Controlled Trials. J. Anxiety Disord. 61, 27-36. doi:10.1016/j.janxdis.2018.08.003

Creamer, M., Foran, J., and Bell, R. (1995). The Beck Anxiety Inventory in a Nonclinical Sample. Behav. Res. Ther. 33, 477-485. doi:10.1016/0005-7967(94) 00082-U

Foa, E. B., and Kozak, M. J. (1986). Emotional Processing of Fear: Exposure to Corrective Information. Psychol. Bull. 99, 20-35. doi:10.1037/0033-2909.99. 1.20

Hamm, A. O. (2020). Fear, Anxiety, and Their Disorders from the Perspective of Psychophysiology. Psychophysiology 57, e13474. doi:10. $1111 /$ psyp. 13474

Ma, X., Yue, Z.-Q., Gong, Z.-Q., Zhang, H., Duan, N.-Y., Shi, Y.-T., et al. (2017). The Effect of Diaphragmatic Breathing on Attention, Negative Affect and Stress in Healthy Adults. Front. Psychol. 8, 874. doi:10.3389/fpsyg.2017.00874

MIND (2021). How Can Nature Benefit My Mental Health? Stratford, London, United Kingdom: MIND.

Naor, L., and Mayseless, O. (2021). Therapeutic Factors in Nature-Based Therapies: Unraveling the Therapeutic Benefits of Integrating Nature in Psychotherapy. Psychotherapy 58, 576-590. doi:10.1037/ pst0000396

Penninx, B. W., Pine, D. S., Holmes, E. A., and Reif, A. (2021). Anxiety Disorders. The Lancet 397, 914-927. doi:10.1016/S0140-6736(21)00359-7
Rothbaum, B. O., Hodges, L. F., Kooper, R., Opdyke, D., Williford, J. S., and North, M. (1995). Effectiveness of Computer-Generated (Virtual Reality) Graded Exposure in the Treatment of Acrophobia. Ajp 152, 626-628. doi:10.1176/ ajp.152.4.626

Standen, P., Threapleton, K., Richardson, A., Connell, L., Brown, D., Battersby, S., et al. (2017). A Low Cost Virtual Reality System for home Based Rehabilitation of the Arm Following Stroke: A Randomised Controlled Feasibility Trial. Clin. Rehabil. 31, 340-350. doi:10.1177/0269215516640320

Zainal, N. H., Chan, W. W., Saxena, A. P., Taylor, C. B., and Newman, M. G. (2021). Pilot Randomized Trial of Self-Guided Virtual Reality Exposure Therapy for Social Anxiety Disorder. Behav. Res. Ther. 147, 103984. doi:10. 1016/j.brat.2021.103984

Conflict of Interest: The authors declare that the research was conducted in the absence of any commercial or financial relationships that could be construed as a potential conflict of interest.

Publisher's Note: All claims expressed in this article are solely those of the authors and do not necessarily represent those of their affiliated organizations, or those of the publisher, the editors and the reviewers. Any product that may be evaluated in this article, or claim that may be made by its manufacturer, is not guaranteed or endorsed by the publisher.

Copyright $\odot 2022$ Premkumar, Heym, Anderson, Brown and Sumich. This is an open-access article distributed under the terms of the Creative Commons Attribution License (CC BY). The use, distribution or reproduction in other forums is permitted, provided the original author(s) and the copyright owner(s) are credited and that the original publication in this journal is cited, in accordance with accepted academic practice. No use, distribution or reproduction is permitted which does not comply with these terms. 